\title{
PREDATOR DIVERSITY AND IDENTITY DRIVE INTERACTION STRENGTH AND TROPHIC CASCADES IN A FOOD WEB
}

\author{
Sonja B. Otto, ${ }^{1,6}$ Eric L. Berlow, ${ }^{1,2,3,5}$ Nathan E. Rank, ${ }^{4}$ John Smiley,${ }^{5}$ and Ulrich Brose ${ }^{1,3}$ \\ ${ }^{1}$ Darmstadt University of Technology, Department of Biology, Schnittspahnstr. 10, 64287 Darmstadt, Germany \\ ${ }^{2}$ University of California, Merced, Sierra Nevada Research Institute, Wawona Station, \\ Yosemite National Park, California 95389 USA \\ ${ }^{3}$ Pacific Ecoinformatics and Computational Ecology Lab, Berkeley, California 94703 USA \\ ${ }^{4}$ Department of Biology, Sonoma State University, Rohnert Park, California 94928 USA \\ ${ }^{5}$ White Mountain Research Station, University of California, Bishop, California 93514 USA
}

\begin{abstract}
Declining predator diversity may drastically affect the biomass and productivity of herbivores and plants. Understanding how changes in predator diversity can propagate through food webs to alter ecosystem function is one of the most challenging ecological research topics today. We studied the effects of predator removal in a simple natural food web in the Sierra Nevada mountains of California (USA). By excluding the predators of the third trophic level of a food web in a full-factorial design, we monitored cascading effects of varying predator diversity and composition on the herbivorous beetle Chrysomela aeneicollis and the willow Salix orestera, which compose the first and second trophic levels of the food web. Decreasing predator diversity increased herbivore biomass and survivorship, and consequently increased the amount of plant biomass consumed via a trophic cascade. Despite this simple linear mean effect of diversity on the strength of the trophic cascade, we found additivity, compensation, and interference in the effects of multiple predators on herbivores and plants. Herbivore survivorship and predator-prey interaction strengths varied with predator diversity, predator identity, and the identity of coexisting predators. Additive effects of predators on herbivores and plants may have been driven by temporal niche separation, whereas compensatory effects and interference occurred among predators with a similar phenology. Together, these results suggest that while the general trends of diversity effects may appear linear and additive, other information about species identity was required to predict the effects of removing individual predators. In a community that is not temporally wellmixed, predator traits such as phenology may help predict impacts of species loss on other species. Information about predator natural history and food web structure may help explain variation in predator diversity effects on trophic cascades and ecosystem function.
\end{abstract}

Key words: biodiversity; Chrysomela aeneicollis; ecosystem function; ecosystem services; food chain; networks; population dynamics; predator-prey; productivity; Salix orestera; trophic interactions.

\section{INTRODUCTION}

A key challenge of environmental biology is to understand how biodiversity loss influences ecosystem function (reviewed by Hooper et al. 2005). Most of the early seminal studies of the relationship between biodiversity and ecosystem function focused on plant diversity and plant productivity (Tilman 1996, 1997, Hooper and Vitousek 1997). However, some studies suggest that high trophic level species such as top predators may be more vulnerable to extinction than species at lower trophic levels (e.g., Pimm et al. 1988, Petchey et al. 1999). Initial theoretical and empirical studies suggest that the relationship between diversity and ecosystem function is more complex and variable at higher trophic levels (Thebault and Loreau 2003, Worm and Duffy 2003, Petchey et al. 2004, Hooper et al. 2005).

Manuscript received 12 January 2007; revised 27 April 2007; accepted 23 May 2007. Corresponding Editor E. D. McCoy.

66-mail: sonotto@bio.tu-darmstadt.de
Thus, it is critical to understand how biodiversity loss at higher trophic levels may propagate through a web of species interactions to influence ecosystem function (Bascompte et al. 2005, Myers et al. 2007). One promising approach for addressing this challenge is to integrate subdisciplines of studies that focus on effects of diversity, per se, on ecosystem function with those that focus on predator-prey dynamics and interaction strengths (Ives et al. 2005, Wootton and Emmerson 2005).

We focus here on three mechanisms proposed by Ives (2005) which regulate how predator diversity can influence both ecosystem function and predator-prey interaction strengths. First, the "sampling effect" or "selection probability effect" occurs when one species of a predator guild dominates effects of that guild on the prey. Thus, effects of predator diversity on prey biomass may be driven primarily by whether one strong (or "keystone") predator is present (e.g., Navarrete and Menge 1996). In this case, predator identity drives the effect of predator diversity on prey biomass and potential cascading effects on plant biomass production. 
Second, additive effects of different predators on a prey species are likely when exploitative competition among predators is weak, because each of them occupies a different niche (Chang 1996, Snyder and Ives 2003) or targets different life history stages of the prey. This is similar to the notion of "complementarity" among plants in resource use, which would cause a monotonic increase in productivity with increased plant diversity (e.g., Tilman et al. 1996). If additive effects of individual predators are of similar strength, effects of predators on shared prey and cascading effects on plant biomass production should depend on the number of predators, but be independent of their identity. Third, multiple predators can have "redundant" or "compensatory" effects on their prey if removal of one is compensated by increases in prey consumption by others (Navarrete and Menge 1996). This is similar to the "insurance hypothesis" (e.g., Tilman 1996, Yachi and Loreau 1999), where functional "redundancy" among species with high niche overlap increases stability of ecosystem function (Walker 1992, Naeem and Li 1997, Tilman 1999). Compensatory effects of predators would suggest that effects of predator removal on prey biomass and plant production should be small until the last predator is removed, irrespective of its identity.

Most experimental studies of predator diversity effects on ecosystem function in complex food webs have been restricted to microcosms (Naeem and Li 1997, 1998, Gamfeldt et al. 2005) or "food web compartments" which were studied in mesocosm enclosures (Petchey and Gaston 2002, Schmitz and Sokol-Hessner 2002, Cardinale et al. 2003, Finke and Denno 2004, 2005, Snyder et al. 2006, Straub and Snyder 2006). Most of these studies also do not incorporate a full factorial manipulation of all predators (but see Schmitz and Sokol-Hessner 2002 for an exception) and thus do not quantify nonadditive interactions among all natural predators. Similarly, these enclosure studies cannot examine interactions among predators with different phenologies. Here, we carry out a full-factorial removal of all predators at the third trophic level in a simple terrestrial food web in the Sierra Nevada mountains of California (USA). This is, to our knowledge, the first full-factorial removal experiment of an entire trophic level under natural field conditions. We quantified prey survival over time and explored how predator-herbivore interaction strengths and potentially nonadditive predator effects govern how consumer diversity influences both herbivore and plant biomass. Finally, by examining individual effects of each predator on beetle survivorship and biomass, we distinguish between effects of (1) consumer identity (i.e., sampling effect), (2) consumer additivity, and (3) consumer compensation.

\section{Materials And Methods}

Field site and food web

The experimental units were located at the South Fork of Bishop Creek $(2860 \mathrm{~m})$ in the Sierra Nevada range in eastern California $\left(37^{\circ} 18^{\prime} \mathrm{N}, 118^{\circ} 56^{\prime} \mathrm{W}\right)$. Experiments were conducted from 23 June 2005, shortly after snow melt, to 10 August 2005. Manipulations were carried out on the willow Salix orestera C. K. Schneid, which occurs along creeks and in bogs from 2800-3500 m altitude (Smiley and Rank 1991). We quantified separate and interactive effects of the three main predator groups (Smiley and Rank 1986) that were most abundant and fed frequently on larvae of the willow leaf beetle Chrysomela aeneicollis Schaeffer (Coleoptera: Chrysomelidae; see Plate 1). We quantified their effects on beetle survivorship and biomass, as well as on the amount of willow leaf tissue consumed by the beetles. The beetles lay egg clutches on the lower surface of willow leaves. After hatching, Chrysomela larvae feed on Salix leaf tissue for three instars and then migrate to the tips of willow shoots to pupate. In the experimental area, predators on these beetles include crawling predators such as a predatory red mite (Prostigmata: Holotrombidae), ants belonging to the genera Formica and Camponotus, a syrphid fly Parasyrphus melanderi Curran (Diptera: Syrphidae), and a solitary wasp Symmorphus cristatus (Vespidae: Eumenidae). As it was impossible to exclude crawling predators individually, and as they consume the same stages of the beetles, we aggregated them as a functional predator type and will subsequently refer to them as "crawlers." Syrphid flies and wasps are specialized predators that consume exclusively P. melanderi larvae. The "crawlers," syrphid flies, and wasps have different phenologies and feeding niches: they occur during different time periods, and they feed on different larval stages. The "crawlers" prey on eggs and first instar larvae of the beetles. Female syrphid flies lay one to nine eggs on young beetle egg clutches. Between hatching and pupation, syrphid larvae feed on eggs and first to third instar beetle larvae (Rank and Smiley 1994). The wasps appear later in the season and prey only on third instar beetle larvae (Sears et al. 2001).

\section{Experimental treatments and response variables}

On 23 June 2005, we applied three different exclusion methods in a full-factorial design to branches that each contained one beetle egg clutch. While there was some natural variation in the number of eggs per clutch $($ clutch size $=43.1 \pm 4.8$ eggs; mean $\pm \mathrm{SD})$, there was no significant difference among treatments in initial egg number (ANOVA, $F_{21,50}=1.27, P=0.24$ ). In each treatment, one or more predators were excluded, which yielded eight treatments with all combinations of "crawlers," syrphid flies, and wasps excluded vs. not excluded. We used one individual branch with one beetle egg clutch per willow clone as an experimental unit (replicate) to maximize replication across host plant individuals. With nine replicates per treatment, a total of 72 experimental units on 72 different willow clones were under observation. We isolated manipulated branches from neighboring branches by clipping surrounding 
foliage to avoid the migration of beetle larvae or crawling predators. Only newly laid beetle egg clutches (discernable by color and smooth surface) were included in experimental branches, which minimized variation in the time exposed to predators.

Crawling predators were excluded by Tanglefoot (Tanglefoot, Grand Rapids, Michigan, USA), a sticky preparation of castor oil and natural gum resins that was applied at the base of observed branches and thus prevented access of mites and ants. Flying predators were excluded by enclosing branches in mesh bags that allowed access by crawling predators. To exclude syrphid flies, we prevented females from ovipositing on beetle egg clutches by enclosing branches in a mesh bag until beetle larvae reached the second instar. These bags were removed in treatments where wasps were not to be excluded before larvae reached the third instar, which was when they were vulnerable to wasp predation. To exclude the wasps, branches were enclosed in mesh bags before beetle larvae reached the third instar. All treatments were applied under natural conditions without affecting densities of the other predator species, beetle densities, or the proportions of different beetle larval stages in the treatments.

We monitored total beetle abundance regularly from 24 June to 9 August 2005. At each monitoring day, we calculated beetle survivorship as the percentage of surviving beetle individuals relative to the initial number of eggs of each clutch. At the end of the experiment, we collected all surviving beetle individuals and measured their total biomass for each replicate. In treatments without mesh bags, some beetle adults left the branches before being collected, which resulted in a number of pupal skins that exceeded the number of adult beetles. In these few cases, we multiplied the number of excess pupal skins by the averaged beetle mass of hatched individuals $(19.4 \pm 3.7 \mathrm{mg}$, mean $\pm \mathrm{SD}, n=76$ new adults) and added this value to the beetle biomass on this branch. This introduced a small error by overestimating total beetle biomass in treatments with smaller than average larvae and underestimating total beetle biomass in treatments with larger than average larvae. To evaluate whether this error affected the results, all analyses were carried out with both beetle biomass and proportional survivorship as response variables.

We calculated the average survivorship of herbivores during the experiment by an average survivorship index (Breden and Wade 1985, Rank 1994). It ranges from zero to one and measures the area beneath the survival curve of all individuals through the time of the whole experiment, divided by the total area if all initial larvae had survived to the last count (Breden and Wade 1987, Rank 1994). In contrast to final beetle number, this variable accounts for beetle survival during the entire time course of the experiment. In particular, it avoids problems with parametric statistical analyses (heteroscedasticity) that emerge when a high proportion of zeros are present in the data. For instance, in 32 of our replicates, all beetles were consumed by the end of the experiment and the final beetle biomass equaled zero.

To quantify the biomass of plant tissue eaten, we collected all leaves of the replicates which showed signs of consumption on 9 August. We used a grid-count method $\left(0.5-\mathrm{cm}^{2}\right.$ cell size) to estimate the foliage area eaten, $f$. We weighted the leaf samples with leaf area, $T$, measured their biomass, $B_{T}$, and calculated the biomass of leaf tissue eaten $\left(B_{t}\right.$; in grams) as $B_{t}=\left(B_{T} / T\right) \times f$.

\section{Statistical analyses and interaction strengths}

To distinguish differences in effects of each predator and combination of predators on beetle survival in time we used repeated-measures ANOVA, with each predator combination as a grouping factor (or interaction among grouping factors) and proportion survival as the dependent variable. To evaluate the significance of predator effects at the end of our experiments, we used $\log _{10}$-transformed beetle biomass, $\log _{10}$-transformed plant biomass eaten, and average survivorship of the beetles as dependent variables. With standard leastsquares three-way full-factorial ANOVAs we tested for effects of predator removal treatments on beetle biomass and survivorship (percentage of initial egg number) at the end of the experiment. In linear least-square regressions, we determined the impact of predator diversity on the dependent variables. Additionally, we carried out ANCOVAs with $\log _{10}$ (beetle biomass) or average beetle survivorship as dependent variable, predator diversity as a continuous predictor, and the presence or absence of the three predators as categorical independent variables. Significant effects of the predator presence-absence variables indicate predator identity effects.

To disentangle the effects of predator diversity from those of changes in total predator density, we compared our measured response variables ( $\log _{10}$ (beetle biomass), $\log _{10}$ (plant biomass eaten), and average survivorship of the beetles) for predator diversity levels of two and three to expected values based on extrapolations of the effects of each predator group alone. The expected effects of multiple predators, $\sigma_{(i+j)}$, were calculated as $\sigma_{(i+j)}=\sigma_{i} \times$ $\sigma_{j} / \sigma_{\mathrm{NP}}$, where $\sigma_{i}$ is the proportion of the effect of one predator on each response variable, $\sigma_{j}$ is the proportion of the effect of a second predator, and $\sigma_{N P}$ is the value of the response variable when no predators are present (Vonesh and Osenberg 2003). An expected value within the standard error of the empirically measured effects indicates additivity, whereas an expected value outside the standard error indicates nonadditivity of multiple predators.

The Interaction Strength (IS) between a predator and the beetles was calculated as the $\log _{10}$ ratio between beetle biomass, $B$, in the presence and absence of the predator: $\mathrm{IS}_{\text {pred } / \text { prey }}=\log _{10}\left(B^{+ \text {pred }} / B^{- \text {pred }}\right)$. We distinguished interaction strengths between any predator and the beetles, depending on predator diversity level and the identity of coexisting predators. In a community of three predator species (e.g., $i, j, k$ ), the interaction strengths 
between, for example, predator $i$ and the beetles can be measured unambiguously at predator diversity levels of one (predator $i$ present, $B^{+i,-j,-k}$ vs. no predator present, $B^{-i,-j,-k}$ ) and three (all three predators present, $B^{+i,+j,+k}$, vs. only the two other predators present, $B^{-i,+j,+k}$ ). At a predator diversity level of two, however, two different interaction strengths describe the effects of predator $i$ on the beetles depending on whether it coexists with predator $j$ (predators $i$ and $j$ present, $B^{+i,+j,-k}$, vs. only predator $j$ present, $B^{-i,+j,-k}$ ) or whether it coexists with predator $k$ (predators $i$ and $k$ present, $B^{+i,-j,+k}$, vs. only predator $k$ present, $B^{-i,-j+k}$ ). These calculations allow analyses of variation in pairwise interaction strengths depending on the identity of coexisting predators. The means and standard errors of interaction strengths at predator diversity levels of one and three were calculated by bootstrapping (2000 random samples of the original data set). We used an ANOVA to test for significant differences among the interaction strengths. The additivity hypothesis predicts that the interaction strength of a predator is independent of the number and identity of the coexisting predators and thus should be similar for the treatments. The redundancy hypothesis predicts that the interaction strength of a predator at the diversity level of one should be substantially higher than its interaction strengths at higher diversity levels, due to compensatory effects of other predators. A positive interaction strength of predator $i$ when coexisting with predator $j$ indicates interference between predators $i$ and $j$, resulting in a higher prey biomass when both predators coexist, compared to treatments without $i$.

\section{Results}

Beetle survival over time in treatments with total predator exclusion (Fig. 1, solid line) was significantly higher than in control treatments without predator removal (natural conditions, Fig. 1, dashed line; repeated-measures ANOVA, $F_{1,16}=22.38, P=0.0002$ ). A significant mortality effect of predators on beetles, i.e., the difference between the bold and dashed lines in Fig. 1 , was observed early in the season (day 7 , repeatedmeasures ANOVA, $P=0.044$ ) and increased during the experiment. At the end of the experiment, the survivorship of the beetles in the treatment without any predators was $36.9 \% \pm 10.2 \%$ (mean $\pm \mathrm{SE}$ ), but survivorship was $1.1 \% \pm 0.9 \%$ on control branches that were exposed to all predators (Fig. 1).

Overall, ANOVAs showed significant effects of the predator removal treatments on $\log _{10}$-transformed beetle biomass $\left(F_{7,63}=2.75, P=0.015\right)$ and beetle survivorship $\left(F_{7,63}=3.53, P=0.003\right)$. In both ANOVAs, we found significant interaction terms between "crawlers" and syrphid flies (for $\log _{10}$-transformed beetle biomass, $P=0.033$; for beetle survivorship, $P=0.01$ ) and a significant main effect of the wasps (for $\log _{10^{-}}$ transformed beetle biomass, $P=0.046$; for beetle survivorship, $P=0.015$ ), whereas all other two-way and three-way interaction terms were not significant. In

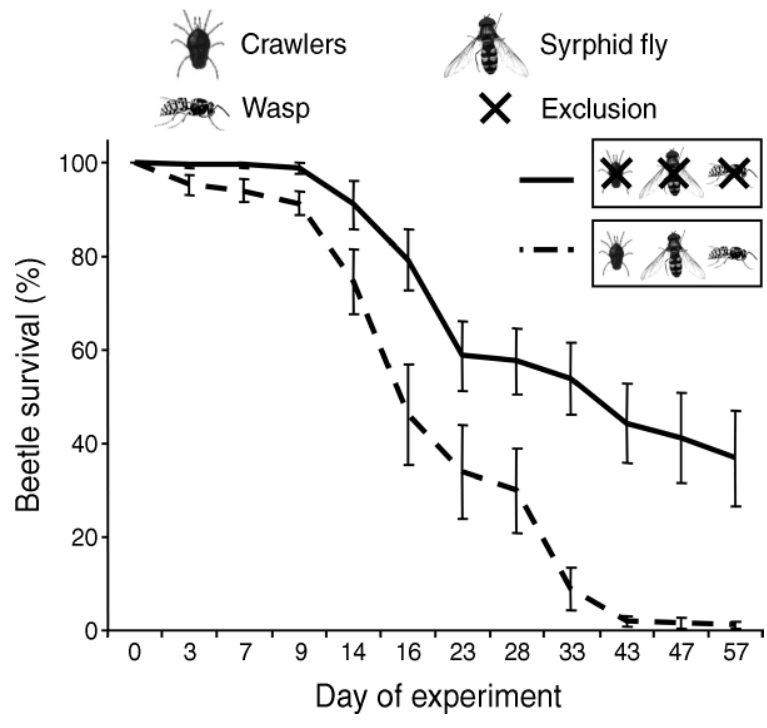

FIG. 1. Time course of willow leaf beetle (Chrysomela aeneicollis) survival (percentage of initial egg number, mean \pm $\mathrm{SE}$ ) from day 0 (experimental setup) to day 57 (sampling and counting of hatched beetle adults): comparison of treatments with all three predators (dashed line) and with total predator exclusion (solid line). The $x$-axis depicts data on sampling dates rather than over a continuous time course. Curves differ significantly (repeated-measures ANOVA: $F_{1,16}=22.38, P=$ $0.0002)$

treatments with single predators (predator diversity level of one), beetle survival was lower (Fig. 2a-c, dashed lines) than under total predator exclusion (Fig. 2a-c, solid lines). The gray bars parallel to the $x$-axis in Fig. $2 \mathrm{a}-\mathrm{c}$ indicate the approximate time periods of the presence of the different predators in the food web (hereafter, phenology) over the course of our study. Thus, the "crawlers" (Fig. 2a) appear earlier in the season than the syrphid flies (Fig. 2b), but then the presence of both predators overlaps considerably, whereas the wasps enter the system later and overlap only for approximately two weeks with the syrphid flies (Fig. 2c). Each predator had a stronger effect when it was the only predator in the food web (Fig. 2a-c) than when it was removed from an intact community with all predators present (Fig. 2d-f). When individual predator treatments were compared to total predator exclusions, "crawlers" and syrphid flies significantly reduced beetle larvae survival over time (Fig. 2a, b; see legend for RM [repeated-measures] ANOVA results). The wasps also reduced the survival of the beetle larvae, but this effect was not statistically significant (Fig. 2c; see legend for RM-ANOVA results). When individual predator removals were compared to unmanipulated treatments (i.e., all predators present), none of the predators had significant effects on beetle survival over the time course of the experiment (Fig. 2d-f, see legend for RMANOVA results). However, in contrast to the other two predators, the effects of wasp removal were marginally significant on the last three sampling dates 

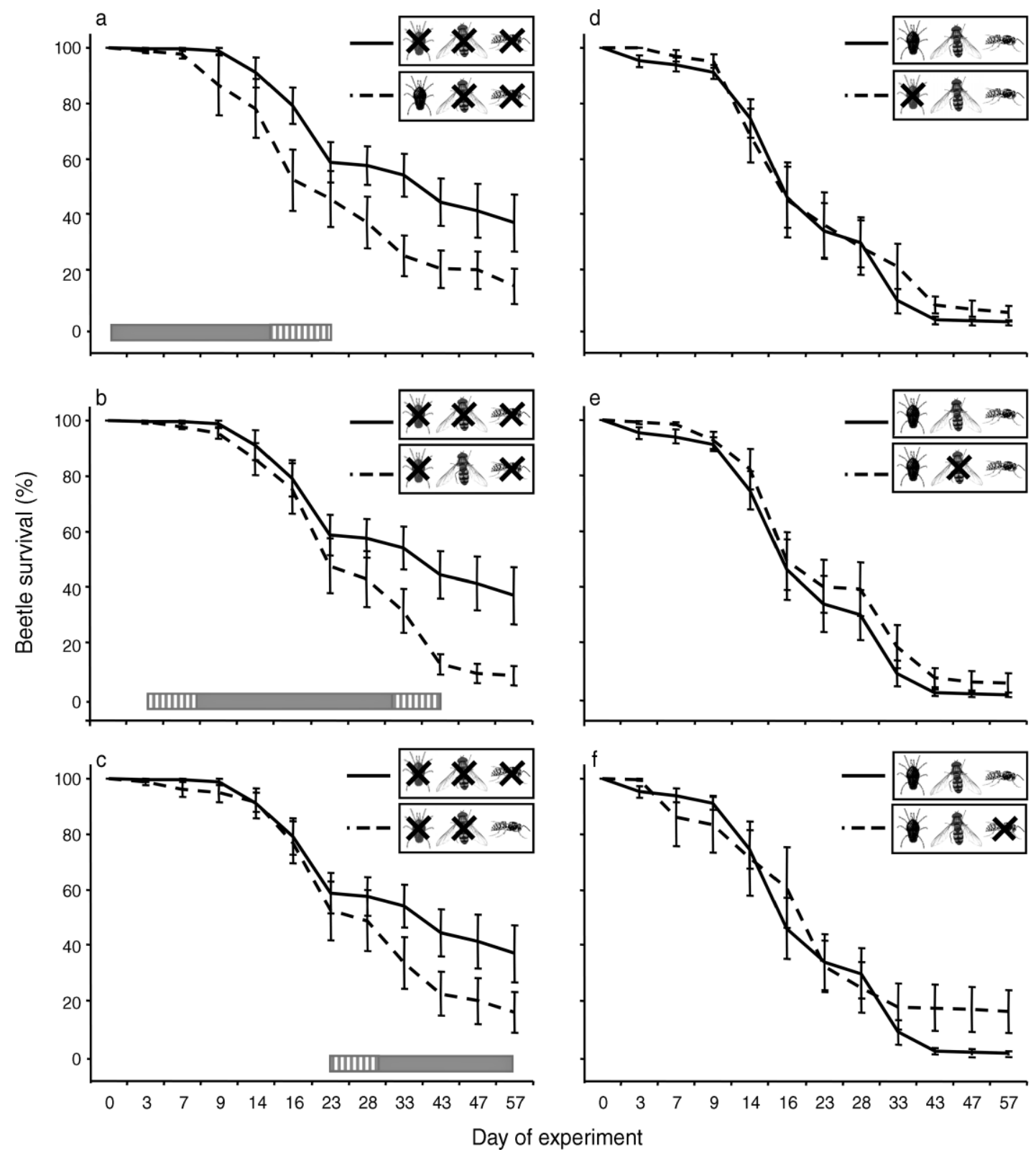

FIG. 2. Time course of beetle survival (percentage of initial egg number, mean \pm SE) from day 0 (experimental setup) to day 57 (sampling and counting of hatched beetle adults). The left-hand panels show the comparison of total predator exclusion (solid lines) vs. single-predator presence (dashed lines) of (a) "crawlers," (b) syrphid flies, or (c) wasps (see Fig. 1 for symbol explanation). The right-hand panels show comparison of the control treatment (no predator removed, solid lines) vs. single-predator exclusion (dashed lines) of (d) "crawlers," (e) syrphid flies, or (f) wasps. Curves in (a) and (b) differ significantly; curves in (c)-(f) do not (repeated-measures ANOVA, (a) $F_{1,16}=5.28, P=0.04$; (b) $F_{1,16}=6.90, P=0.02$; (c) $F_{1,16}=2.25, P=0.15$; (d) $F_{1,16}=0.21, P=0.65$; (e) $F_{1,16}=1.12, P=0.31$; (f) $F_{1,16}=0.31, P=0.59$ ) (ANOVA on last day of experiment: (a) $P=0.077$, (b) $P=0.017$, (c) $P=0.114$, (d) $P=0.152$, (e) $P=0.269$, (f) $P=0.067$ ). The $x$-axis depicts data on sampling dates rather than over a continuous time course. The gray horizontal bars indicate the phenology of the predators in the food web; dashed ends indicate that phenology is not bound to strict dates.

(Fig. 2f, see legend for last day). In general, the trends in Fig. 2 suggest ecologically significant predator effects on beetle survivorship that are consistent with the predator's appearance in the food web: When each predator is considered in isolation (Fig. 2a-c), the effect of "crawlers" on beetle survival magnified after day 9 (late egg stage and some first instar larvae, Fig. 2a), the effect of syrphid flies on beetles became distinct after day 23 (most syrphid maggots hatched at the time of late first instar and early second instar beetle larvae; Fig. 2b), and the trend for wasp effects on beetles is largest after day 28 (late second and early third-instar larvae, Fig. 2c). 


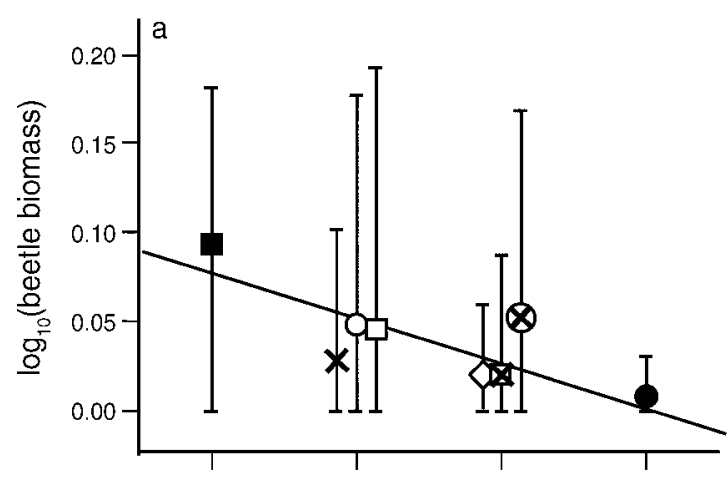

Predators present

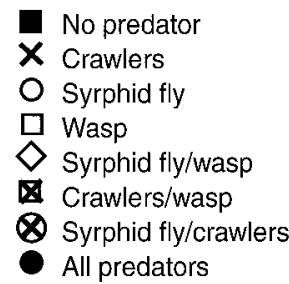

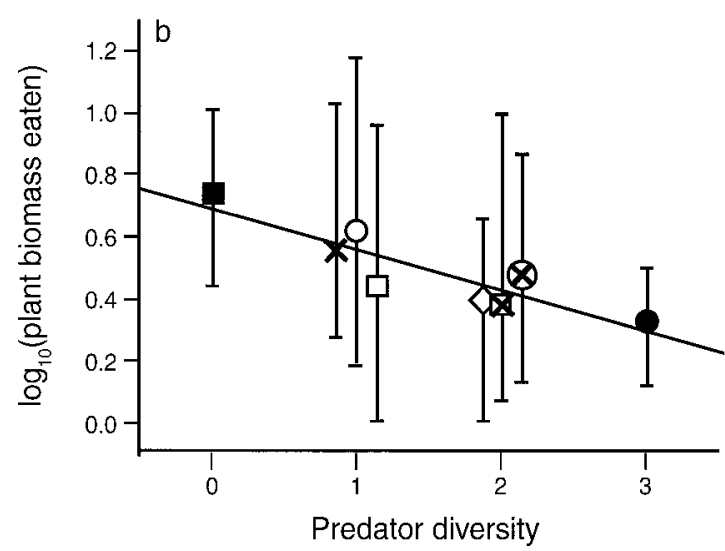

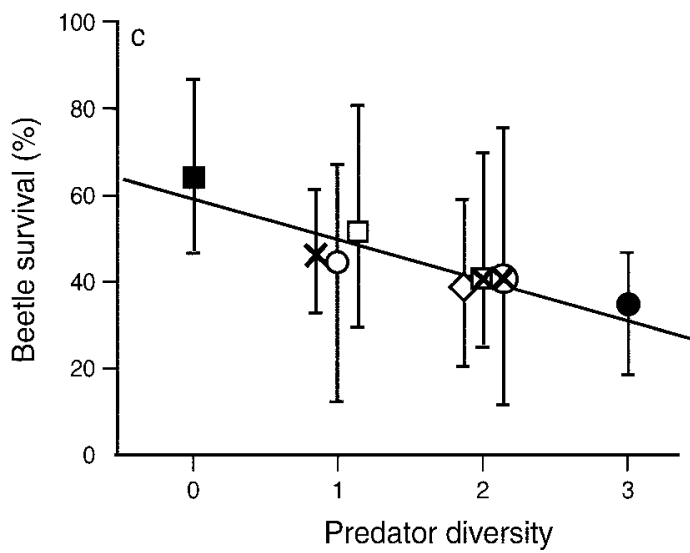

FIG. 3. Predator diversity ( $0-3$ species) effects on (a) $\log _{10}$-transformed beetle biomass (originally measured in grams); (b) the amount of $\log _{10}$-transformed plant biomass consumed by the beetles (originally measured in grams); and (c) the average survivorship of beetle individuals at the end of the experiment. Data columns are offset (at predator diversity levels of 1 and 2) by 0.02 for better determination of predator identity effects. Symbols show treatment means; whiskers indicate the treatment minima and maxima. Regression details follow $( \pm \mathrm{SE})$ : (a) $y=0.077( \pm 0.01)-0.025( \pm 0.007) x, R^{2}=0.16, F_{1,69}=13.35, P=0.0005$; (b) $y=$ $0.69( \pm 0.07)-0.13( \pm 0.04) x, R^{2}=0.15, F_{2,57}=10.66, P=0.0018 ;(\mathrm{c}) y=0.59( \pm 0.03)-0.09( \pm 0.02) x, R^{2}=0.22, F_{2,67}=19.40, P<$ 0.0001 .

Increasing predator diversity caused a significant decrease in (1) $\log _{10}$-transformed beetle biomass (Fig. 3a), (2) $\log _{10}$-transformed plant biomass consumed (Fig. $3 b$ ), and (3) average survivorship of the beetles (Fig. 3c). While these central tendencies were highly significant $(P$ $<0.002$ in all cases), the variance within treatments was substantial $\left(R^{2}<0.22\right)$. A three-species predator community reduced the biomass of the beetles by 96.15\% compared to the all-predator exclusion treatments. Increasing predator diversity also increased the strength of a trophic cascade through the food web to significantly decrease the amount of plant biomass consumed (Fig. 3b). The complete predator community reduced the amount of plant biomass consumed by $75 \%$ compared to replicates where all predators were removed from the food web. Increasing predator diversity from zero to three reduced the absolute amount of plant biomass consumed $(3.75 \mathrm{~g}) 15$ times more than it reduced beetle biomass $(0.25 \mathrm{~g})$. The effects of individual predators on the amount of plant biomass removed (Fig. 3b) were similar to those on beetle biomass (Fig. 3a). The exception to this pattern was that "crawlers," as single predators at a predator diversity level of one, reduced beetle biomass more than the wasps (Fig. 3a), whereas the wasps reduced the amount of plant biomass consumed more strongly than the "crawlers" (Fig. 3b).

To evaluate whether the observed effect of increased predator diversity (Fig. 3a-c) could be explained by increased predator density alone, we compared the observed effect to that expected based on extrapolations of the individual predator effects. Expected beetle biomasses at predator diversities of two and three lay within one standard error of the mean observed values, except for the treatment where syrphid flies and "crawlers" coexisted $\left(\log _{10}\right.$-transformed beetle biomass mean $[ \pm \mathrm{SE}]$, expected $=0.0139$, observed $=0.052$ $[ \pm 0.02])$. The slope of the regression through the expected values $(-0.028 \pm 0.005 \mathrm{SE})$ is within one standard error of the regression slope through the observed values (regression details in legend to Fig. 3a). The expected values for plant biomass eaten and the slope of the regression (slope $=-0.16$ ) are within one standard error of the observed values. The expected values for the average survivorship of the beetles are all within the standard errors of the observed values except 


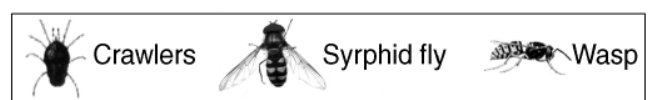

a) Predator diversity $=1$ or 3

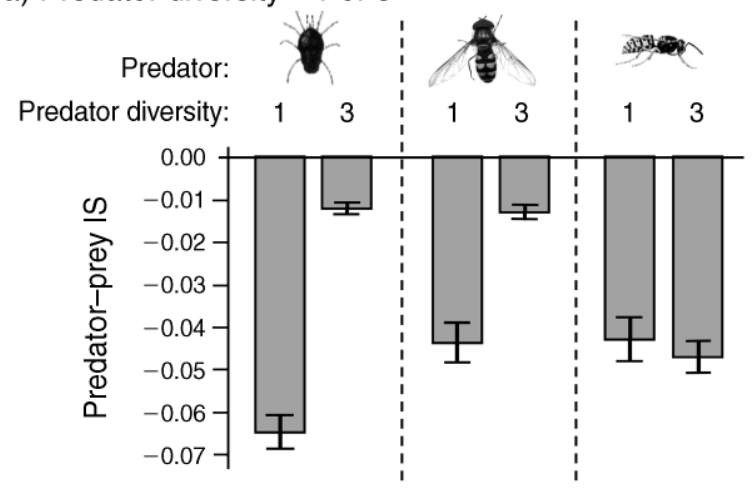

b) Predator diversity $=2$

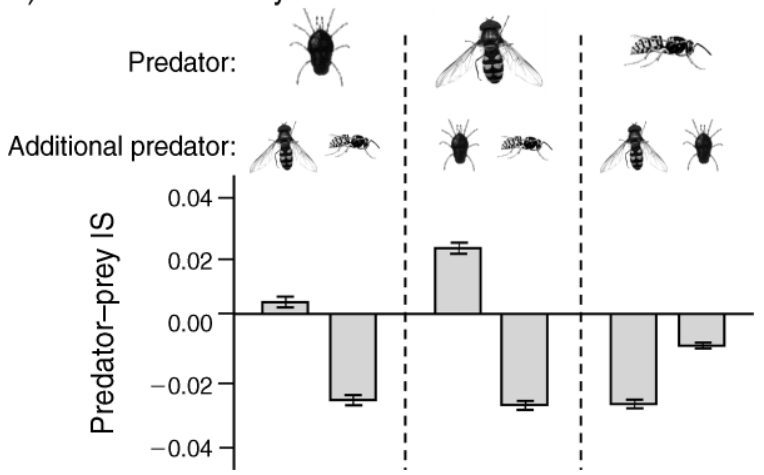

FIG. 4. Predator-prey interaction strength (IS, mean $\pm \mathrm{SE}$, bootstrapped data set; for calculation of IS see Materials and methods: Statistical analyses and interaction strengths) measured in terms of beetle biomass at the end of the observation period. (a) Pairwise predator-prey IS at predator diversity levels of 1 (individual effects) and 3 (effect of predator removal from "intact" community); one-way ANOVA $F_{5,1994}=32.4, P<$ 0.0001 . (b) Pairwise predator-prey IS at a predator diversity level of 2. Each IS for each predator depends on the identity of the coexisting predator. Here, the different IS may be of similar or different algebraic signs, indicating additive (negative values) or inhibitory interactions (positive values) between the predators.

for a higher than expected survival in the treatment where all predators are present (expected $=0.29$, observed $[ \pm \mathrm{SE}]=0.35[ \pm 0.03])$. The slope of the relationship between expected survivorship and predator diversity was marginally steeper than the observed slope (slope expected, -0.11 , slope observed $[ \pm \mathrm{SE}]-0.09$ [ \pm 0.02$])$.

To disentangle the effects of predator diversity and predator identity, we used an analysis of covariance with average beetle survivorship and $\log _{10}$ beetle biomass at the end of the experiments as response variables. These analyses suggest that the predator effects we observed were driven by predator diversity but not the identity of present predators (beetle biomass, $P=0.002$ and 0.33 for predator diversity and predator identity, respectively; average survivorship, $P=0.0004$ and 0.56 for predator diversity and predator identity, respectively.). Similar results were obtained with a type-I ANOVA, in which predator diversity was entered before predator identity.

Predator-prey interaction strengths (IS) varied with predator diversity (Fig. 4a) and with the identity of coexisting predators (Fig. 4b). The subsequent results describe trends in biomass-based interaction strengths, but qualitatively identical trends characterize interaction strengths analyses based on beetle survivorship at the end of the experiment. All predators had a strong effect on beetle biomass when no other predators co-occurred (predator diversity level equals one, Fig. 4a) and the effect of "crawlers" was significantly stronger than the effects of syrphid flies or wasps (Tukey's hsd test, $P<$ 0.05 ). When all three predators were present, however, wasp removal had a significantly stronger impact on beetle biomass than the removal of either "crawlers" or syrphid flies alone (Tukey's hsd test, $P<0.05$; Fig. 4a). The effect of the wasps on beetle biomass was similar at the predator diversity levels of one (i.e., single-predator community) and three (i.e., an "intact" community with all three predators; Fig. 4a). In contrast, the "crawlers" and syrphid flies had significantly stronger effects on the beetles in the single-predator treatments than in the "intact" community of three predators (Tukey's hsd test, $P<0.05$; Fig. 4a). When predators were removed from a two-predator community, the magnitude of their effects on beetles depended on the identity of the coexisting predator (Fig. 4b). If the wasps coexisted with "crawlers" or syrphid flies, all effects on beetles were negative. However, if "crawlers" and syrphid flies coexisted in the food web, beetle biomass was on average higher in the presence of both predators than in treatments in which one of the predators had been removed (Fig. 4b), indicating predator interference.

Average body mass of beetle individuals $(y)$ decreased significantly with increasing beetle abundance $(A)$ at the end of the experiments (ordinary linear least square regression $[ \pm \mathrm{SE}]: y=0.022( \pm 0.0008)-0.0002( \pm 0.00006) A ; R^{2}$ $=0.22, P=0.003)$, increasing beetle biomass $(B$; linear least square regression $( \pm \mathrm{SE}): y=0.021( \pm 0.0008)-$ $\left.0.008( \pm 0.004) B ; R^{2}=0.13, P=0.025\right)$, and thus with decreasing predator diversity ( $D$; linear least square regression $( \pm \mathrm{SE}): y=0.017( \pm 0.0009)-0.002( \pm 0.0006) D$; $R^{2}=0.19, P=0.006$ ).

\section{Discussion}

In this study, increasing predator diversity significantly decreased herbivore biomass and average survivorship, which subsequently decreased the amount of plant biomass consumed via a trophic cascade. We did not find evidence that predator identity or a "sampling effect" drove these predator diversity effects on prey populations. Given the simplicity of the food web in our study and the trophic similarity of the predators, we hypothesized that the loss of any one of them would be 


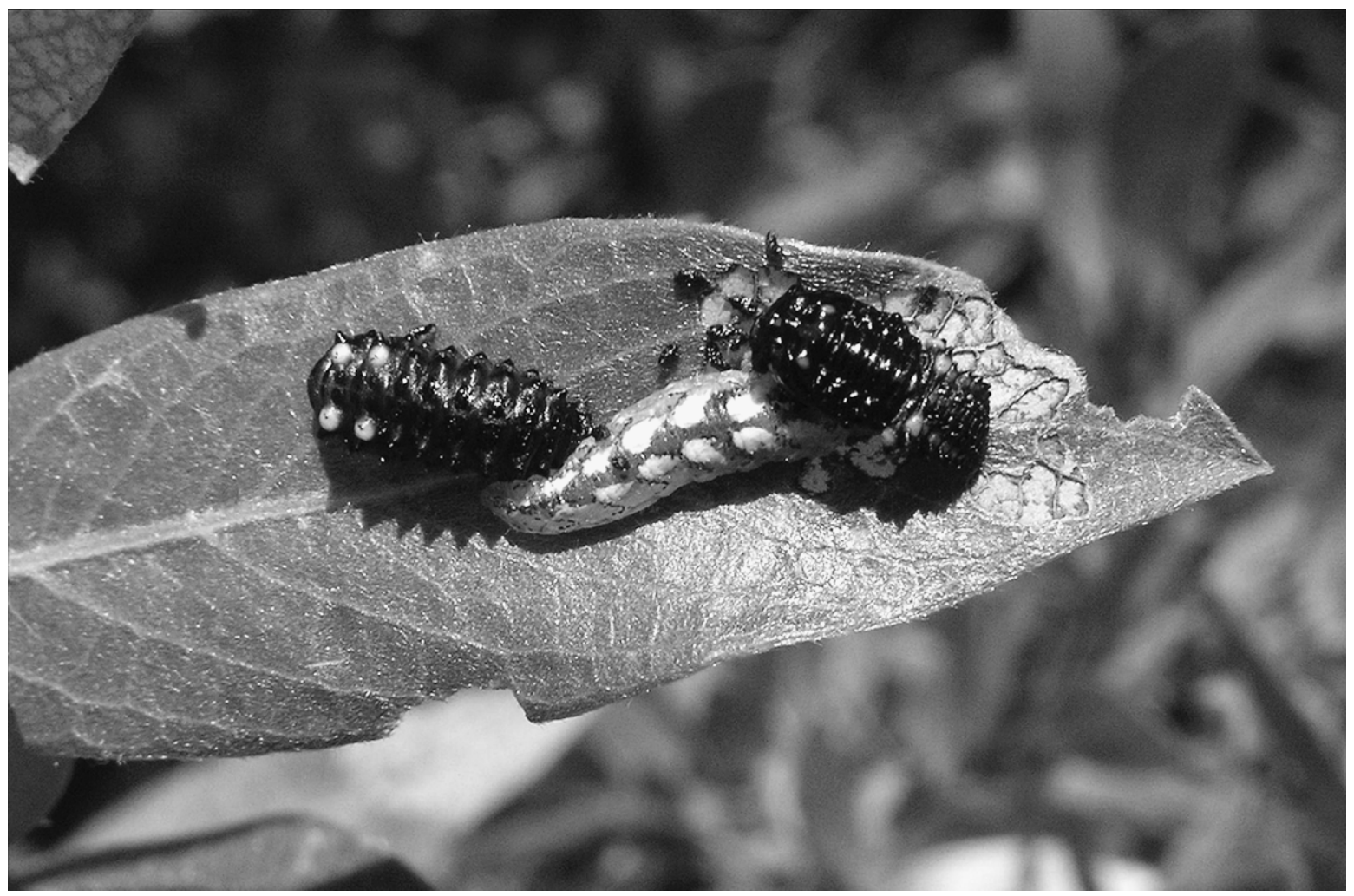

Plate 1. The herbivore willow leaf beetle (Chrysomela aeneicollis) feeds on the willow Salix orestera at high elevations in California. Shown are: (left, black with white dots) one third-instar larvae of the beetle and (right) one pupa. Feeding damage on the willow is visible on the right edge of the leaf. (Center) Larvae of the hover fly Parasyrphus melanderi (pale with white spots) feed exclusively on these beetles at montane field sites. Photo credit: S. B. Otto.

compensated for by the others. However, our analyses of pair-wise interaction strengths suggest that the effects of wasps on beetles was similar if it was the only predator or if it was removed from an intact predator community (diversity level 3), whereas effects were weaker when it was removed from a two predator community. This result is caused by the significant interference between the other two predators, syrphid flies and "crawlers." The beetle biomass in the treatment with only syrphid flies and "crawlers" was much higher than in the other two-predator communities. Therefore, removing the wasp from the intact three-predator community led to a strong increase in beetle biomass, an effect which was as strong as the wasp effect when removed from a single-predator community. While effects were strong for all three predators when alone, interference between syrphids and "crawlers" also meant that the removal of either one from an intact predator community had much weaker effects than their removal when alone because the other one was released from interference. The removal of wasps from a two-predator community had a weaker effect compared to its removal from the three-predator community, because either the syrphids or the "crawlers" exerted a strong effect on the beetles prior to the wasps' appearance. Thus, strong interference between two predators (e.g., syrphids and crawlers) generated variable effects of predator diversity on pair-wise interaction strengths. Interestingly, these strong nonadditive interactions combined to create an overall mean diversity effect which appeared additive.

As one possible mechanism for the described patterns, we propose that distinct predator phenologies determine the potential for additivity and interference among species through their influence on both temporal overlap among predators and the larval stages upon which they feed. Wasps appear later in the season than either syrphid flies or crawling predators, and they feed exclusively on third-instar beetle larvae, which increases the likelihood that their effect will be additive. Crawlers and syrphid flies have more temporal and dietary overlap with respect to beetle larval development, which may explain their significant interference and their ability to reciprocally compensate for the loss of the other. The exclusion of one of these two species thus leads to a release of interference by the other species. Both processes, the release of interference and compensatory increases in population consumption, cause the lack of a removal effect when the other species is present. This interpretation of our results suggests that the predator identity effects on interaction strengths are driven by the phenology of predators, which results in a temporal niche separation. However, due to the low 
number of predator species in this community, predator identity and phenology could not be disentangled in our experiment. In particular, the wasps were the only predators with a late phenology, and other traits of the wasps that could influence the effect of their identity on interaction strengths were thus confounded with phenology. Our hypothesis, that predator phenology determines additive, compensatory, and interference effects needs to be tested in a community with more predator species of varying phenology.

Our results suggest that predator diversity and identity drive interaction strengths and trophic cascades when populations are not temporally well mixed. Interestingly, extrapolations of the single-species treatment effects to higher levels of predator diversity yielded expected values that were in most cases consistent with the observed values. While this could be interpreted to mean that the observed predator diversity effect was the result of changing total predator density across diversity treatments, our analyses of interaction strengths point to a combination of additivity, compensation, and interference among predators, which renders this explanation unlikely. Instead, it is likely that the variability caused by species-specific effects of predator diversity on interaction strengths generated an overall trend that appears additive. Nevertheless, we manipulated predator diversity in a field setting where all non-excluded species were allowed to vary naturally in order to explicitly investigate the consequences of biodiversity loss under natural conditions. Therefore, we cannot entirely rule out potential confounding effects of predator abundance changing with diversity levels.

Although we found a consistently strong negative effect of predator diversity on the mean herbivore biomass and the amount of plant biomass consumed, the variance among replicates was substantial. This high within-treatment variance, in effect strength, was most likely caused by the random spatial variation of detection of prey and predator nesting sites. Thus, not every trophic interaction is realized at every spatial location (Brose et al. 2004), which lowers average interaction strengths but increases their spatial variance (Berlow 1999).

Increased variance at lower predator diversity could be due to the "sampling effect" (Ives et al. 2005), where one dominant predator has a strong effect, regardless of the number of coexisting predators. This hypothesis predicts that the variance in effect strength should be low in treatments in which a species with the dominant effect on herbivore or plant biomass reduction is present or absent across all replicates (Navarrete and Menge 1996). In our study system, no predator species had a dominant effect on herbivore or plant biomass reduction - a result which is in contrast to many prior studies on predator-diversity effects on ecosystem functioning (Finke and Denno 2005, Gamfeldt et al. 2005, Straub and Snyder 2006). Although wasps effects were additive to other predators, they did not have a dominant effect on the beetles. This was due to the fact that wasp effects were strong in a three-predator community but not stronger than effects of other predators in the singlepredator or two-predator communities. Thus we conclude that predator identity did not drive the relationship between predator diversity and beetle biomass, survivorship, or herbivory.

Changes in species diversity at higher trophic levels may propagate through a food web via trophic cascades (Schmitz et al. 2000, Paine 2002, Shurin et al. 2002, Schmitz 2003). The loss of predators leads to higher herbivore abundance and thus lower plant biomass (Halaj and Wise 2001, Cardinale et al. 2003, Byrnes et al. 2006) or plant productivity (Carpenter et al. 1985, Duffy et al. 2003, Finke and Denno 2004). Yet, Shurin (2002) showed that many terrestrial trophic cascades are dampened at the herbivore-plant interface. Surprisingly, our results suggest a magnification of effects as they propagate down the food chain: when comparing the exclusion of all predators vs. the presence of all predators, the average reduction of plant biomass consumed was fifteen times larger than the reduction of beetle biomass. This may be explained by several facts. First, for building one unit of their own biomass, herbivores have to consume more than twice the amount of plant biomass, because their assimilation efficiency is less than 50\% (Yodzis and Innes 1992). Second, the total plant biomass consumed includes effects of beetles that fed early and then were consumed by predators. The biomass of these beetle individuals is not included in the biomass of beetles recorded at the end of the experiment. Third, the magnification of cascading effects may be due to trait-mediated effects such that beetle larvae may feed less in the presence of predators (Krivan and Schmitz 2004, Schmitz et al. 2004, Byrnes et al. 2006). Our finding of a comparatively strong trophic cascade is consistent with recent suggestions that trophic cascades are more likely in communities with invertebrate herbivores that lack intraguild predation and in habitats with high resource availability (Borer et al. 2005, Finke and Denno 2005).

The effect strength of a predator on plant biomass via a trophic cascade was correlated with interaction strength of the predator on the herbivorous beetles. The only exception to this pattern is that "crawlers" as single predators have a stronger effect on beetle biomass than on amount of plant biomass consumed, whereas the wasps showed the opposite pattern. The crawlers consume beetle larvae of the first instar mainly. Beetles that escape predation by "crawlers" grow 5-50 times larger than the early instars and consequently reduce plant biomass most severely during their third instar. Additionally, we found evidence for compensatory growth of beetle individuals that escape predation. In contrast to the crawlers, the wasps prey on late second or third instar beetle larvae and thus remove individuals with the highest maximum consumption rates. Together 
these facts may explain the difference between crawler effects on beetles and plants in our experiments.

\section{Conclusions}

Empirical and theoretical studies have shown that interaction strengths between species depend on species composition of the community (Berlow 1999, Brose et al. 2005). Therefore, the ability of any given predator to compensate for the loss of another predator depends on the composition of the remaining predator community (Straub and Snyder 2006). Consistent with these results, we found that pairwise predator-prey interaction strengths varied with predator diversity and the identity of the coexisting predators. Despite clear interference and compensation among individual predators, we observed an overall linear mean effect of predator diversity on herbivore biomass and herbivore consumption of plants. The patterns of additivity or redundancy in individual predator effects may have been driven by the phenological appearance of each predator in the food web. Interference and compensatory effects occurred between predators with a similar feeding niche, while temporally distinct predator populations may have prevented early season predators from compensating for the removal of late season predators. Thus, while predator diversity has an overall linear effect on the strength of the trophic cascade, other information about species identity was required to predict the effects of removing individual predators. If these findings generalize to more diverse food webs, phenological information along with knowledge on the food web structure (Bascompte et al. 2005, Brose et al. 2005) and species' body sizes (Emmerson and Raffaelli 2004, Brose et al. $2006 a, b)$ might facilitate predictions of the consequences of predator loss in complex ecosystems.

\section{ACKNOWLEDGMents}

We are grateful to the staff of the White Mountain Research Station, University of California, USA (WMRS) for help with the logistics of work and stay of S. B. Otto in California. Comments and suggestions by two anonymous referees, Elizabeth Dahlhoff, and Stefan Scheu are appreciated and have greatly improved this study. Financial support has been provided to U. Brose and S. B. Otto by the German Research Foundation (BR 2315/1-1,2; BR 2315/4-1), to S. B. Otto by the WMRS and the "Advancement of Women in Science" fellowship of the Technical University of Darmstadt, and to E. L. Berlow by the Humboldt Foundation.

\section{REFERENCES}

Bascompte, J., C. J. Melian, and E. Sala. 2005. Interaction strength combinations and the overfishing of a marine food web. Proceedings of the National Academy of Sciences (USA) 102:5443-5447.

Berlow, E. L. 1999. Strong effects of weak interactions in ecological communities. Nature 398:330-334.

Borer, E. T., E. W. Seabloom, J. B. Shurin, K. E. Anderson, C. A. Blanchette, B. Broitman, S. D. Cooper, and B. S. Halpern. 2005. What determines the strength of a trophic cascade? Ecology 86:528-537.

Breden, F., and M. Wade. 1985. The effect of group size and cannibalism rate on larval growth and survivorship in Plagiodera versicolora. Entomography 3:455-463.
Breden, F., and M. J. Wade. 1987. An experimental study of the effect of group size on larval survivorship in the imported willow leaf beetle Plagiodera versicolora (Coleoptera: Chrysomelidae). Environmental Entomology 16:1082-1086.

Brose, U., E. L. Berlow, and N. D. Martinez. 2005. Scaling up keystone effects from simple to complex ecological networks. Ecology Letters 8:1317-1325.

Brose, U., et al. 2006a. Consumer-resource body-size relationships in natural food webs. Ecology 87:2411-2417.

Brose, U., A. Ostling, K. Harrison, and N. D. Martinez. 2004. Unified spatial scaling of species and their trophic interactions. Nature 428:167-171.

Brose, U., R. J. Williams, and N. D. Martinez. $2006 b$. Allometric scaling enhances stability in complex food webs. Ecology Letters 9:1228-1236.

Byrnes, J., J. J. Stachowicz, K. M. Hultgren, A. R. Hughes, S. V. Olyarnik, and C. S. Thornbert. 2006. Predator diversity strengthens trophic cascades in kelp forests by modifying herbivore behavior. Ecology Letters 9:61-71.

Cardinale, B. J., C. T. Harvey, K. Gross, and A. R. Ives. 2003. Biodiversity and biocontrol: emergent impacts of a multipleenemy assemblage on pest suppression and crop yield in an agroecosystem. Ecology Letters 6:857-865.

Carpenter, S. R., J. F. Kitchell, and J. R. Hodgson. 1985. Cascading trophic interactions and lake productivity. BioScience 35:634-638.

Chang, G. C. 1996. Comparison of single versus multiple species of generalist predators for biological control. Environmental Entomology 25:207-212.

Duffy, J. E., J. P. Richardson, and E. A. Canuel. 2003. Grazer diversity effects on ecosystem functioning in seagrass beds. Ecology Letters 6:637-645.

Emmerson, M. C., and D. Raffaelli. 2004. Predator-prey body size, interaction strength and the stability of a real food web. Journal of Animal Ecology 73:399-409.

Finke, D. L., and R. F. Denno. 2004. Predator diversity dampens trophic cascades. Nature 429:407-410.

Finke, D. L., and R. F. Denno. 2005. Predator diversity and the functioning of ecosystems: the role of intraguild predation in dampening trophic cascades. Ecology Letters 8:1299-1306.

Gamfeldt, L., H. Hillebrand, and P. R. Jonsson. 2005. Species richness changes across two trophic levels simultaneously affect prey and consumer biomass. Ecology Letters 8:696-703.

Halaj, J., and D. H. Wise. 2001. Terrestrial trophic cascades: how much do they trickle? American Naturalist 157:262-281.

Hooper, D. U., et al. 2005. Effects of biodiversity on ecosystem functioning: a consensus of current knowledge. Ecological Monographs 75:3-35.

Hooper, D. U., and P. M. Vitousek. 1997. The effects of plant composition and diversity on ecosystem processes. Science 277:1302-1305.

Ives, A. R., B. J. Cardinale, and W. E. Snyder. 2005. A synthesis of subdisciplines: predator-prey interactions, and biodiversity and ecosystem functioning. Ecology Letters 8: 102-116.

Krivan, V., and O. J. Schmitz. 2004. Trait and density mediated indirect interactions in simple food webs. Oikos 107:239-250.

Myers, R. A., J. K. Baum, T. D. Shepherd, S. P. Powers, and C. H. Peterson. 2007. Cascading effects of the loss of apex predatory sharks from a coastal ocean. Science 315:18461850.

Naeem, S., and S. B. Li. 1997. Biodiversity enhances ecosystem reliability. Nature 390:507-509.

Naeem, S., and S. B. Li. 1998. Consumer species richness and autotrophic biomass. Ecology 79:2603-2615.

Navarrete, S. A., and B. A. Menge. 1996. Keystone predation and interaction strength: interactive effects of predators on their main prey. Ecological Monographs 66:409-429.

Paine, R. T. 2002. Trophic control of production in a rocky intertidal community. Science 296:736-739. 
Petchey, O. L., A. L. Downing, G. G. Mittelbach, L. Persson, C. F. Steiner, P. H. Warren, and G. Woodward. 2004. Species loss and the structure and functioning of multitrophic aquatic systems. Oikos 104:467-478.

Petchey, O. L., and K. J. Gaston. 2002. Extinction and the loss of functional diversity. Proceedings of the Royal Society of London Series B 269:1721-1727.

Petchey, O. L., P. T. McPhearson, T. M. Casey, and P. J. Morin. 1999. Environmental warming alters food-web structure and ecosystem function. Nature 402:69-72.

Pimm, S. L., H. L. Jones, and J. Diamond. 1988. On the risk of extinction. American Naturalist 132:757-785.

Rank, N. E. 1994. Host-plant effects on larval survival of a salicin-using leaf beetle Chrysomela aeneicollis Schaeffer (Coleoptera: Chrysomelidae). Oecologia 97:342-353.

Rank, N. E., and J. T. Smiley. 1994. Host-plant effects on Parasyrphus melanderi (Diptera, Syrphidae) feeding on a Willow Leaf Beetle Chrysomela aeneicollis (Coleoptera, Chrysomelidae). Ecological Entomology 19:31-38.

Schmitz, O. J. 2003. Top predator control of plant biodiversity and productivity in an old-field ecosystem. Ecology Letters 6: 156-163.

Schmitz, O. J., P. A. Hamback, and A. P. Beckerman. 2000. Trophic cascades in terrestrial systems: a review of the effects of carnivore removals on plants. American Naturalist 155: 141-153.

Schmitz, O. J., V. Krivan, and O. Ovadia. 2004. Trophic cascades: the primacy of trait-mediated indirect interactions. Ecology Letters 7:153-163.

Schmitz, O. J., and L. Sokol-Hessner. 2002. Linearity in the aggregate effects of multiple predators in a food web. Ecology Letters 5:168-172.

Sears, A. L. W., J. T. Smiley, M. Hilker, F. Muller, and N. E. Rank. 2001. Nesting behavior and prey use in two geographically separated populations of the specialist wasp Symmorphus cristatus (Vespidae: Eumeninae). American Midland Naturalist 145:233-246.

Shurin, J. B., E. T. Borer, E. W. Seabloom, K. Anderson, C. A. Blanchette, B. Broitman, S. D. Cooper, and B. S. Halpern. 2002. A cross-ecosystem comparison of the strength of trophic cascades. Ecology Letters 5:785-791.

Smiley, J. T., and N. E. Rank. 1986. Predator protection versus rapid growth in a montane leaf beetle. Oecologia 70:106-112.

Smiley, J. T., and N. E. Rank. 1991. Bitterness of Salix along the north fork of Big Pine Creek, eastern California: species and community elevational trends. Pages $132-147$ in C. A.
Hall, V. Doyle-Jones, and B. Widawski, editors. Natural history of eastern California and high-altitude research, University of California. White Mountain Research Station Symposium. The Regents of the University of California, Los Angeles, California, USA.

Snyder, W. E., and A. R. Ives. 2003. Interactions between specialist and generalist natural enemies: parasitoids, predators, and pea aphid biocontrol. Ecology 84:91-107.

Snyder, W. E., G. B. Snyder, D. L. Finke, and C. S. Straub. 2006. Predator biodiversity strengthens herbivore suppression. Ecology Letters 9:789-796.

Straub, C. S., and W. E. Snyder. 2006. Species identity dominates the relationship between predator biodiversity and herbivore suppression. Ecology 87:277-282.

Thebault, E., and M. Loreau. 2003. Food-web constraints on biodiversity-ecosystem functioning relationships. Proceedings of the National Academy of Sciences (USA) 100: 14949-14954.

Tilman, D. 1996. Biodiversity: population versus ecosystem stability. Ecology 77:350-363.

Tilman, D. 1997. Distinguishing between effects of species diversity and species composition. Oikos 80:185.

Tilman, D. 1999. The ecological consequences of changes in biodiversity: a search for general principles. Ecology 80 : 1455-1474.

Tilman, D., D. Wedin, and J. Knops. 1996. Productivity and sustainability influenced by biodiversity in grassland ecosystems. Nature 379:718-720.

Vonesh, J. R., and C. W. Osenberg. 2003. Multi-predator effects across life-history stages: non-additivity of egg- and larval-stage predation in an African treefrog. Ecology Letters 6:503-508.

Walker, B. H. 1992. Biodiversity and ecological redundancy. Conservation Biology 6:18-23.

Wootton, J. T., and M. Emmerson. 2005. Measurement of interaction strength in nature. Annual Review of Ecology, Evolution, and Systematics 36:419-444.

Worm, B., and J. E. Duffy. 2003. Biodiversity, productivity and stability in real food webs. Trends In Ecology and Evolution 18:628-632.

Yachi, S., and M. Loreau. 1999. Biodiversity and ecosystem productivity in a fluctuating environment: the insurance hypothesis. Proceedings of the National Academy of Sciences (USA) 96:1463-1468.

Yodzis, P., and S. Innes. 1992. Body size and consumerresource dynamics. American Naturalist 139:1151-1175. 